\title{
Noise Radiated by an Open Cavity at Low Mach Number: Effect of the Cavity Oscillation Mode
}

\author{
Rocio Martin ${ }^{1,2}$, Manel Soria ${ }^{1}$, Oriol Lehmkuhl ${ }^{3}$, Andrey Gorobets ${ }^{4}$ and Alexey Duben ${ }^{4}$
}

\begin{abstract}
The present work focuses on the study of noise generation and radiation of an infinite open three-dimensional cavity at low Mach number with laminar upstream conditions, that is of interest to understand noise generation mechanisms in wall-bounded separated flows. A particular feature of this configuration is the oscillatory mode: shear layer mode or wake mode. For the parameters considered in the present study it is seen that while in shear layer mode the flow shows a two-dimensional behaviour, in the wake mode the flow is three-dimensional, resulting in significantly different sound sources. The influence of the acoustic feedback mechanism in SL mode has also been investigated comparing the results between different momentum thickness values at the cavity inlet. This paper presents results of sound radiated by a three-dimensional infinite open cavity with aspect ratio $L / D=4$ at Reynolds number based on the cavity depth of $\operatorname{Re}_{D}=1500$ and Mach number of $M=0.15$, both for shear layer $(L / \theta=67)$ and wake $(L / \theta=84)$ oscillation modes. To do so, Curle integral evaluated as a post-process of an incompressible solution will be used. The results are compared with the resulting Curle post-process of a two-dimensional incompressible simulation.
\end{abstract}

\author{
Keywords \\ Computational Aeroacoustics, Low Mach Number, Curle Method, Cavity Modes
}

\section{Introduction}

Aeroacoustics is a topic of research with increasing relevance due to its technological and societal implications. It is of particular interest to the aeronautic industry where continued effort is put on the reduction of noise produced, for instance, by the landing gear $[1 ; 2]$ or the jet $[3 ; 4]$. The automotive industry is also focused on the study of flow past vehicles and the noise induced by it. To mention a few works, $[5 ; 6]$ studied the noise generated by the air flow around the side mirror of a generic vehicle model while [7; 8] investigated the half cylinder and the hemisphere, respectively, as a simplification of side mirrors. Open wide cavities, the object of the present work, are also common parts of vehicle designs and their acoustic performance is a key issue of comfort for passengers or outside listeners. Recently, the noise generated by the rear door gap considered as a wide cavity with a small opening was studied in [9].

As extensively documented in [10], computational aeroacoustics cases can be addressed from two general approaches: Direct Simulation (DS) and hybrid methods. Direct Simulation is based on the resolution of the compressible Navier-Stokes equations without using any modelling of the sound. This method is the most direct way to compute sound generation since the governing equations completely describe the physics of the problem. However, solving the full set of equations implies extreme computational costs, out of the scope for the majority of industrial applications. On the other hand, hybrid approaches seem to be more promising to predict aeroacoustic noise. These consist on two different numerical solvers: an unsteady CFD tool as the source generator and an acoustic solver as the transport method.
This work focuses on integral approaches which mainly rest on the work of Lighthill, [11]. From the computational point of view, integral methods are simpler and allow to have an explicit solution for the acoustic pressure, which has to be numerically evaluated from the source terms obtained in the Navier Stokes solver. Two of the most widely used integral methods are the extension of Lighthill's theory for the interaction of the fluid with a surface at rest, known as Curle's solution [12], and for the interaction of the fluid with a surface in motion, Ffwocs-Williams and Hawkings (FW-H) solution [13].

A broad body of literature on open cavity noise investigation has been developed over the last decade. A significant fraction of the studies are about finite width cavities, that are of interest for aeronautic applications. Among others, Lai and Luo [14] studied the acoustic field of a cavity at $R e_{L}=\frac{\rho_{\infty} U_{\infty} L}{\mu}=1.36 \times 10^{6}$ using the FW$\mathrm{H}$ formulation, with dimensions $L / D=5$ and $W / D=1$, where $L, D$ and $W$ are respectively the length, depth and width of the cavity. Lately, other works such as [15] addressed the same configuration at $\operatorname{Re}_{L}=1.29 \times 10^{7}$ and $R e_{L}=2.2 \times 10^{7}$ for $M=\frac{U_{\infty}}{a_{0}}=0.85$ and $M=1.2$. In [16], the open cavity at $\operatorname{Re}_{\theta}=8300$, where $\theta$ is the momentum thickness of the boundary later at the inlet of

\footnotetext{
${ }^{1}$ Universitat Politècnica de Catalunya, Spain.

${ }^{2}$ SEAT S.A., Spain

${ }^{3}$ Barcelona Supercomputing Center, Spain

${ }^{4}$ Keldysh Institute of Applied Mathematics of RAS, Russia.
}

\section{Corresponding author:}

Manel Soria, Carrer de Colom, 11, 08222 Terrasa, Barcelona, Spain.

Email: manel.soria@upc.edu 
the cavity, and $M=0.12$ was investigated using DS. The geometrical parameters were $L / D=0.5$ and $W / D=0.6$.

The configuration considered here is a very wide open cavity. Some of the first computations of acoustic radiation for this configuration were carried out by Hardin and Pope in [17]. The authors investigated the sound generated by an open cavity with aspect ratio $L / D=4$, at Reynolds number based on the cavity length $R e_{L}=5000$ and Mach number $M=0.1$, where $U_{\infty}$ is the freestream velocity, $\rho_{\infty}$ the freestream density, $\mu$ the dynamic viscosity and $a_{0}$ the sound speed in the fluid. They concluded that the cavity flow undergoes a characteristic regular oscillation which leads to the radiation of sound. The open cavity with the same aspect ratio at $R e_{D}=1500$ and $M=0.15$ was studied by Larsson and Davidson et al. [18]. The authors confirmed that Curle's solution based on a compressible simulation was in good agreement with DS. They also found that the contribution of the wall pressure fluctuations accounted for approximately $90 \%$ of the radiated intensity. Ask and Davidson [19; 20] extended this study evaluating Curle's integral based on an incompressible simulation, also finding acceptable results though being sceptical about the contribution of the outlet wall. The aforementioned works treat the flow as two-dimensional. However, Brès and Colonius [21] numerically showed that for cavities of aspect ratio $L / D=4$, the transition to three-dimensional flow was found to be at $R e_{D} \approx 1200$. This was previously determined by Faure and Lusseyran in an experiment [22]. This value is slightly below the Reynolds number considered in this work, $R e_{D}=\frac{\rho_{\infty} U_{\infty} D}{\mu}=1500$.

Another important aspect of the cavity flow is the oscillation mode. Gharib and Roshko [23], in an experimental work, found that in addition to a non-oscillating mode, there are two different oscillation modes: shear layer (SL) and wake mode (WM). The oscillation mode depends on the development of the boundary layer at the cavity inlet. Later, Colunius, Rowley and Basu [24; 25] used two-dimensional compressible flow equations for cavities with aspect ratios ranging from 1 to 5 and a wide range of $R e_{\theta}$ values, where $\theta$ is the upstream boundary momentum thickness. The transition from SL to WM was obtained as $L / \theta$ or $R e_{\theta}$ increased. The SL mode is characterised by a feedback loop between the acoustics waves travelling upwards and the shedding vortex detached from the leading edge of the cavity [24]. On the other hand, the WM is characterised by a largescale shedding from the cavity leading edge directed into the cavity and later violently ejected from it. While in the SL mode the acoustic waves play an important role for the self-sustained oscillations in the cavity, they are no longer involved in the WM flow [24].

The present work is focused in an infinite open cavity of aspect ratio $L / D=4$ at $R e_{D}=1500$ and $M=0.15$, oscillating in WM $(L / \theta=84)$ and $\operatorname{SL}(L / \theta=67)$. This configuration was already studied in WM by $[18 ; 19 ; 20]$. The main goals have been: (i) confirm that at this Reynolds number, only slightly above the transition, the flow is threedimensional in WM and/or in SL; (ii) study the effects of the three-dimensional structures on the sound generation, using incompressible simulation plus Curle's integral; (iii) study if SL oscillations are obtained without the acoustic feedback mechanism in incompressible simulations and, if so, study their acoustic behaviour.

This paper is organised as follows. First the hybrid method developed by Curle for predicting the acoustic response due to flow past a solid body is described. Secondly, the case under study is presented followed by the description of the numerical methods used to address it. Finally, the results obtained for the flow and acoustic fields are explained. The results include the comparisons between four simulations: two-dimensional (2D) and three-dimensional (3D), each of them with SL and WM (i.e., 2D-SL, 2D-WM, 3D-SL, 3DWM).

\section{Computational Aeroacoustics Method}

Lighthill [11] considered the compressible continuity and momentum equations under no external forces:

$$
\begin{aligned}
\frac{\partial \rho}{\partial t}+\frac{\partial}{\partial x_{i}} \rho u_{i} & =0 \\
\frac{\partial}{\partial t} \rho u_{i}+\frac{\partial}{\partial x_{j}}\left(\rho u_{i} u_{j}+p_{i j}-\tau_{i j}\right) & =0
\end{aligned}
$$

where $x_{i}$ are the spatial coordinates (or $x, y$ and $z$ ), $u_{i}$ are the velocity components (or $u, v$ and $w$ ), $p$ the pressure, $\rho$ the density and $\tau_{i j}$ the viscous stress tensor,

$$
\tau_{i j}=\mu\left(\frac{\partial u_{i}}{\partial x_{j}}+\frac{\partial u_{j}}{\partial x_{i}}-\frac{2}{3} \frac{\partial u_{k}}{\partial x_{k}} \delta_{i j}\right) .
$$

A simple mathematical manipulation of the previous equations lead to the inhomogeneous wave equation,

$$
\frac{\partial^{2} \rho}{\partial t^{2}}-a_{0}^{2} \nabla^{2} \rho=\frac{\partial^{2} T_{i j}}{\partial x_{i} \partial x_{j}}
$$

where $T_{i j}=\rho u_{i} u_{j}+p_{i j}-a_{0}^{2} \rho \delta_{i j}-\tau_{i j}$ is known as the Lighthill's stress tensor. Lighthill [11] showed that it is sufficient to assume $T_{i j}=\rho u_{i} u_{j}+p_{i j}-a_{0}^{2} \rho \delta_{i j}-\tau_{i j} \approx$ $\rho_{0} u_{i} u_{j}$ when considering low Mach numbers, isentropic flow and negligible viscous effects.

The Curle's solution for the Lighthill's formulation is given by:

$$
\begin{aligned}
\rho(\mathbf{x}, t)-\rho_{0} & =\frac{1}{4 \pi a_{0}^{2}} \frac{\partial^{2}}{\partial x_{i} x_{j}} \int_{V} \frac{\left[T_{i j}\right]}{r} d V \\
& +\frac{1}{4 \pi a_{0}^{2}} \frac{\partial}{\partial x_{i}} \int_{S} \frac{n_{j}}{r}\left[p_{i j}-\tau_{i j}\right] d S,
\end{aligned}
$$

where the expression in brackets stands for the evaluation of the expression at the retarded time $\tau=t-r / a_{0}, r$ is the distance between the observer position and the source point and $n$ is the surface normal vector pointing to the surface.

Curle's integral accounts for two type of sources: a quadrupole source arising from the volume integral (first term) and a dipole source arising from the surface integral (second term). Quadrupoles sources are less efficient producing acoustic waves than monopoles or dipoles sources, specially for low Mach numbers. In [12], it was shown that for low Mach number flows the relation between the acoustic power generated by quadrupoles and dipoles is 
related by the Mach number:

$$
\frac{P_{q u a d}}{P_{d i p}} \approx M^{2} .
$$

This justifies that the authors of [20;26], working at $M=0.15$ and $M=0.2$ respectively, considered volumetric sources negligible. In [27], it was numerically confirmed that the contribution of the source terms inside the volume integral for the cavity case at $M=0.15$ were several orders of magnitude smaller than the source terms inside the surface integral.

In order to implement Eq.5 the derivatives are taken inside the integral and spatial derivatives are transformed into time derivatives. This procedure was first done by Myers and Farassat in [28] to modify the solution of Kirchhoff's formula, primarily used in the theory of diffraction of light and in other electromagnetic problems, [29]. Therefore, the final equation to be solved is:

$$
\begin{aligned}
p(\mathbf{x}, t)-p_{0} & =\frac{1}{4 \pi} \int_{V}\left(\frac{l_{i} l_{j}}{a_{0}^{2} r}\left[\ddot{T}_{i j}\right]+\frac{3 l_{i} l_{j}-\delta_{i j}}{a_{0} r^{2}}\left[\dot{T}_{i j}\right]\right. \\
& \left.+\frac{3 l_{i} l_{j}-\delta_{i j}}{r^{3}}\left[T_{i j}\right]\right) d V \\
& +\frac{1}{4 \pi} \int_{S}-l_{i} n_{j}\left(\frac{1}{r a_{0}}\left[\dot{p}_{i j}\right]+\frac{1}{r^{2}}\left[p_{i j}\right]\right) d S
\end{aligned}
$$

where the viscous term has been omitted and assuming isentropy, $\rho$ has been expressed in terms of $p$. The vector $l$ is the unitary vector pointing from the source point to the observational point:

$$
l_{i}=\frac{x_{i}-y_{i}}{r} .
$$

An important advantage of using Curle's formulation when it is reduced to a surface integral is that it provides direct information about the contribution of each point of the surface to the total acoustic power. This can be of much use in the industry since it could highlight the potential parts of the surface to be optimized. In the recent years, techniques based on beamforming analysis combined with an acoustic technique have been used for the localisation of sound sources [30]. However, Curle's formulation is specially interesting when the Mach number is low since it allows an incompressible simulation as a source for the transient flow variables over the solid body. Moreover, this allows to neglect the volume integral which would be computationally expensive and only take into account pressure effects over the surface.

The case of the present study accomplishes that the relative position between the source and the observers is constant and that the Mach number is considered low, so the use of Curle's approach using an incompressible simulation as source terms is justified.

\section{Problem Statement}

The Curle analogy is applied to study the aeroacoustic radiation of an open cavity (sketched in Fig.1), in presence of an upstream laminar flow, using two different flow sources

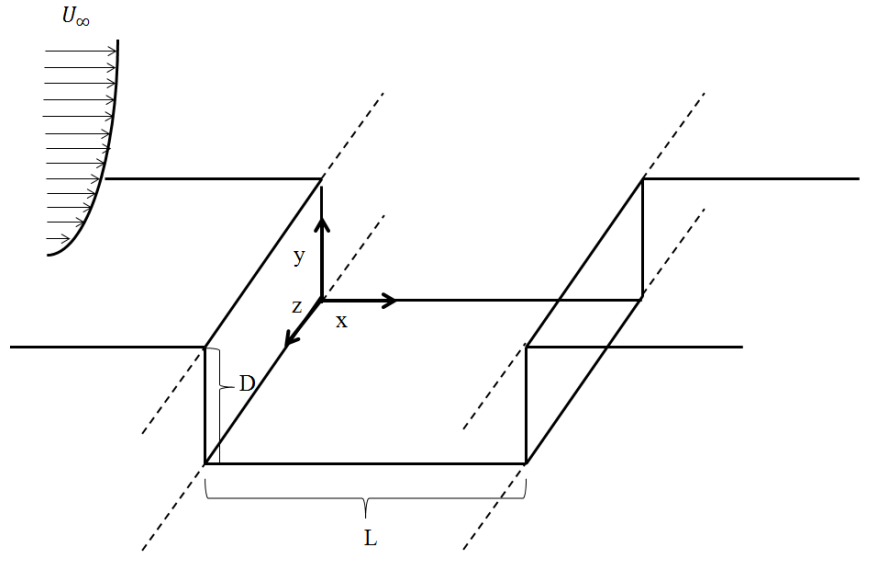

Figure 1. Geometry of the cavity in the present work.

obtained from: i) two-dimensional incompressible simulation and ii) three-dimensional incompressible simulation. In the present case, $R e_{D}=1500$ and the Mach number is $M=0.15$.

As previously explained, the thickness of the boundary layer arriving at the leading edge of the cavity can substantially change the behaviour of the flow around the cavity. Colonius et al. [24] established the transition between shear and wake mode in $L / \theta \approx 75$ for $\operatorname{Re}_{\theta}=58.6$,for both two-dimensional and three-dimensional simulations. The SL runs have been performed with $L / \theta \approx 67\left(R e_{\theta}=89.5\right)$ and the WM runs with $L / \theta \approx 84\left(R e_{\theta}=71.4\right)$. Considering that the boundary layer arriving at the leading edge of the cavity has the profile of the Blasius solution for a flat plate, the momentum thickness is:

$$
\theta=0.664 \sqrt{\frac{\nu x}{U_{\infty}}},
$$

where $\nu$ is the kinematic viscosity and $x$ is the distance downstream from the onset of the boundary layer. In both WM and SL simulations, the domain begins $8 D$ units before the cavity leading edge. Therefore, the WM case is generated imposing a uniform profile at the inlet of the domain, while the Blasius profile developed for $x=4 D$ is imposed for the SL mode.

No slip boundary conditions are used in solid boundaries. Velocity variables are specified by zero normal derivatives and $p=0$ at the outflow boundary. However, in order to damp the two-dimensional vortical structures at the outlet of the domain, a buffer zone is imposed for the last 10 mesh elements. This is not necessary in the three-dimensional simulations. Finally, considering that the cavity is infinite in the spanwise direction, periodic boundary conditions have been imposed at the front and back boundary walls.

\section{Computational Methods}

The CFD code used to solve the governing equations is Alya, the code developed by Barcelona Supercomputing Center, [31]. In the code Alya, the convective term is discretized using a Galerkin FEM scheme recently proposed in [32], which conserves linear/angular momentum and kinetic energy at a discrete level. Neither an upwinding 
scheme nor equivalent momentum stabilization is used. In order to use equal-order elements, numerical dissipation is introduced only for the pressure stabilization via a fractional step scheme [33], which is similar to approaches for pressure-velocity coupling in the unstructured collocated finite volume codes [34]. This approach has been shown to be significantly less dissipative compared to traditional stabilized FEM approach [35]. Temporal discretization is performed through a conservative explicit third-order RungeKutta scheme [36]. Due to the low Reynolds number, no turbulence model has been used, so all the simulations will be considered as DNS.

\section{Computational Domain}

As shown in Figure 4, the computational domain extends over $32 D$ in the $x$-direction, with the leading edge of the cavity located at $8 D$ from the upstream boundary. In the $y$-direction, the height is $8 D$. In the case of the threedimensional simulation the spanwise width is $3 D$. The extension of the cavity in the $z$-direction is long enough to ensure uncorrelated turbulent fluctuations at a separation of half the domain size for the WM. Figure 2 displays the autocorrelation coefficient for the 3D-SL and 3D-WM at a point located in the cavity, $x / D=3$ and $y / D=-0.5$. The autocorrelation coefficient is defined as:

$$
R_{i i}=\frac{<u_{i}^{\prime}\left(x_{i}, t\right) u_{i}^{\prime}\left(x_{i}+\delta, t\right)>}{<u_{i}^{\prime} u_{i}^{\prime}>}
$$

where $\langle *\rangle$ denotes the time average, $u_{i}^{\prime}=u_{i}-\bar{u}_{i}$ the velocity fluctuation and $\delta$ the distance in the spanwise direction. The autocorrelation coefficient tends to zero for the WM as it approaches the half-size of the domain for the three components of the velocity. However, for the SL mode it can be seen that there is a high correlation between points separated by 1 length unit (see also the instantaneous $\mathcal{Q}$ and $p$ in Figures 11 and 15). In both cases, these results confirm that a width of 3 is enough to statistically represent flow variables in the spanwise direction. As an additional check it is important to verify that the domain is sufficiently wide to compute the acoustic results. Figure 3 shows the autocorrelation coefficient of the pressure fluctuation for three points located on the wall: $(x / D, y / D)=(3,-1)$, $(x / D, y / D)=(4,0)$ and $(x / D, y / D)=(5,0)$. The results confirm that the pressure fluctuations are also uncorrelated before reaching half of the domain size and thus $W / D=3$ should be enough for this case.

\section{Computational Grid}

A non-structured grid has been used for the $x-y$ planes. The nodes are clustered near the walls, within the cavity and in the wake region (see Fig.4). The mesh for the three-dimensional case has been created extruding a twodimensional mesh. The number of parallel planes in the $z$ direction is 60 with $\Delta z / D=0.05$. A grid convergence study has been carried out in order to ensure the accuracy of the acoustic results. As an example, the results obtained for the 3D-WM are discussed below. Two different meshes, with $4.06 \times 10^{6}$ nodes (mesh 3D-WM-a) and $5.81 \times 10^{6}$ nodes (mesh 3D-WM-b) have been used.
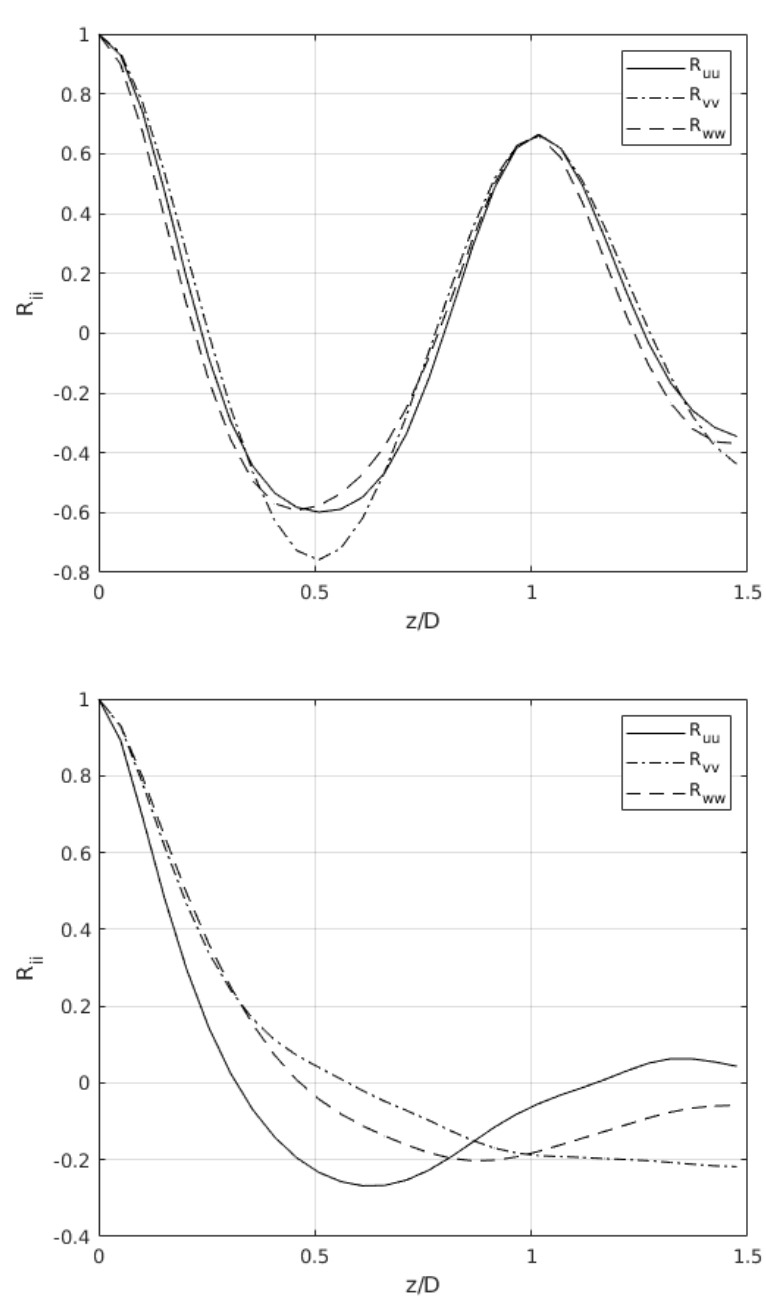

Figure 2. Autocorrelation of velocity fluctuations along the $z$ axis for a probe located at $(x / D, y / D)=(3,-0.5)$. Top: 3D-SL. Bottom: 3D-WM.

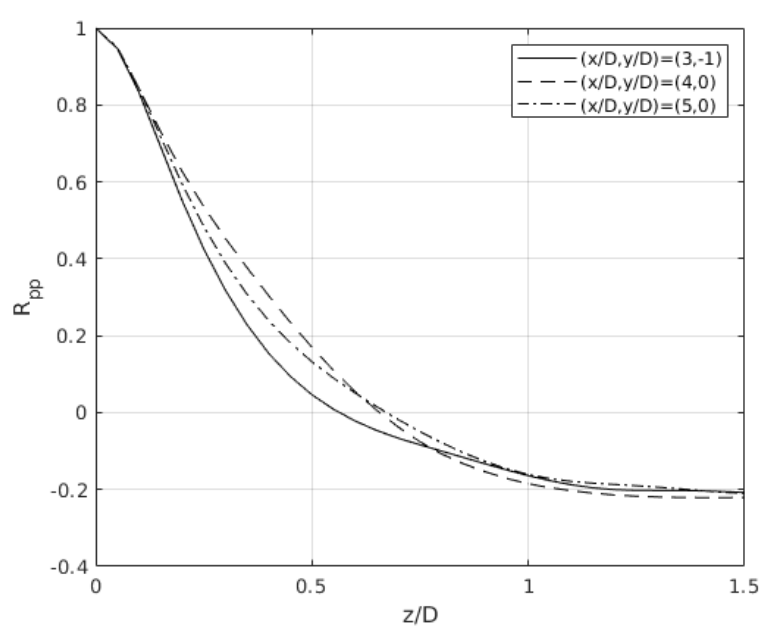

Figure 3. Autocorrelation of pressure fluctuations along the $z$ axis for three probes located at $(x / D, y / D)=(3,-1)$, $(x / D, y / D)=(4,0)$ and $(x / D, y / D)=(5,0)$.

A comparison of the frequency spectra for the pressure history at the cavity trailing edge $(x / D=4, y / D=0)$, the 


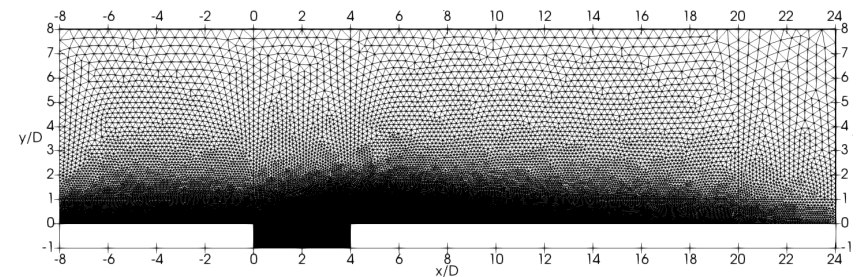

Figure 4. Grid in the $x-y$ plane for 3D-WM-b.

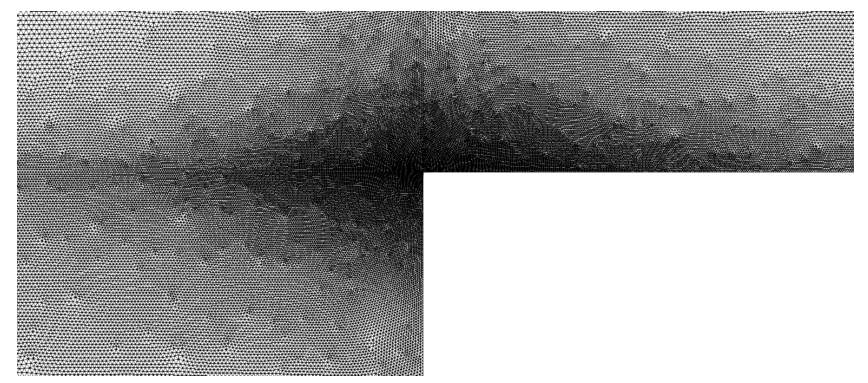

Figure 5. Grid near the cavity 3D-WM-b.

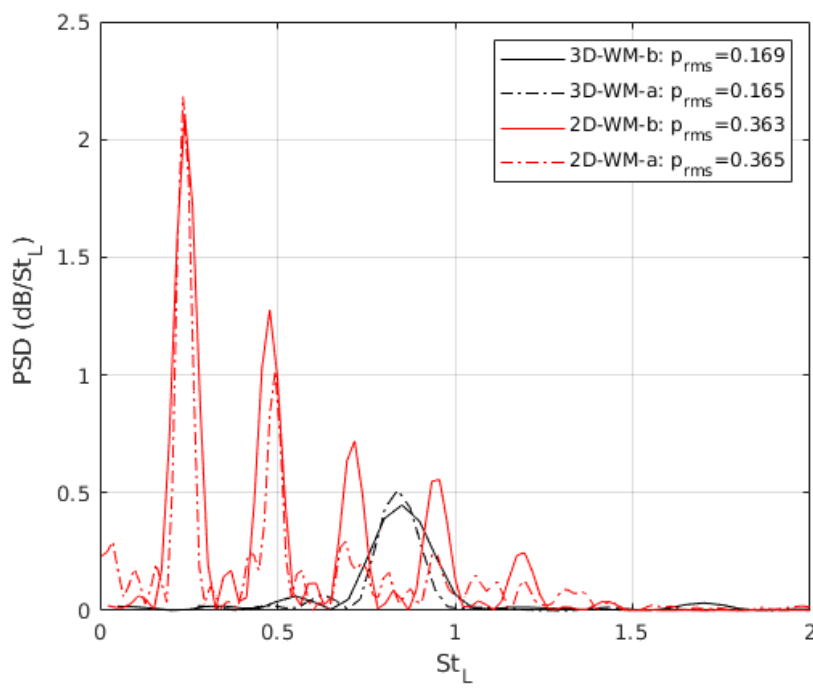

Figure 6. Frequency spectra and $p_{r m s}$ value of $p$ at $(x / D=4$, $y / D=0)$.

point with highest pressure fluctuations, obtained with both meshes is shown in Figure 6. Both, for 3D-WM and 2D$\mathrm{WM}$, the root mean square value of $p, p_{r m s}$, varies less than $2 \%$. The drag coefficient has also been used to analyse the convergence. Table 1 shows the time averaged drag coefficient $C_{D}$ and $S t_{L}$ obtained. The drag coefficient is computed as

$$
C_{D}=\frac{F_{D}}{\frac{1}{2} \rho_{\infty} U_{\infty}^{2} D W}
$$

where $F_{D}$ is the force contribution from the three cavity walls and $W$ is the width of the cavity. The Strouhal number, $S t_{L}$, is defined as:

$$
S t_{L}=\frac{f L}{U_{\infty}}
$$

where $f$ is the fundamental frequency, i.e., the frequency with the highest peak in the power spectrum.

\begin{tabular}{|c|c|c|}
\hline Case & $C_{D}$ & $S t_{L}$ \\
\hline 2D-WM-a & 0.408 & 0.235 \\
\hline 2D-WM-b & 0.403 & 0.232 \\
\hline 3D-WM-a & 0.062 & 0.901 \\
\hline 3D-WM-b & 0.066 & 0.891 \\
\hline
\end{tabular}

Table 1. Time averaged value of drag coefficient and $S t_{L}$ for the mesh convergence study.

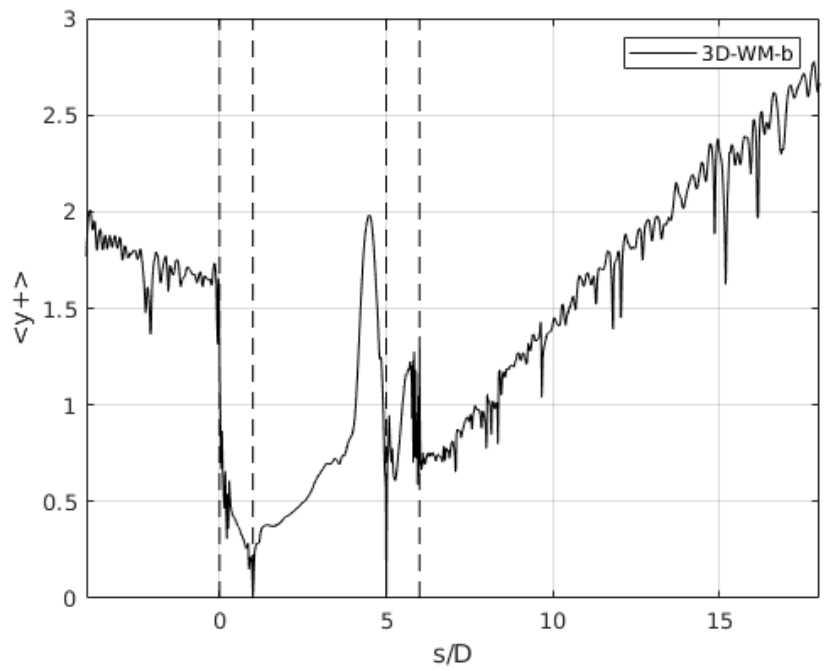

Figure 7. $<y+>$ value along the wall for 3D-WM-b The discontinuous vertical lines correspond to the edges of the cavity.

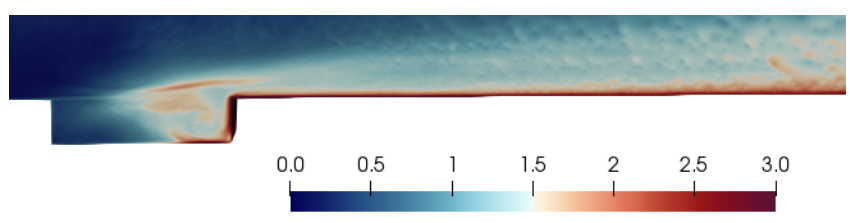

Figure 8. $\Delta / \eta$ ratio for 3D-WM-b.

As can be seen, the differences in $C_{D}$ between both meshes are in the order of $1 \%$ and $6 \%$ for the $2 \mathrm{D}$ and $3 \mathrm{D}$ cases, and about $1 \%$ in both cases for the Strouhal number.

Figure 7 shows the time-averaged value of $y+$ along the wall, obtained from the statistical post-processing of the 3DWM-b results, as a function of $s$ (the distance from the leading edge along the wall). The discontinuous vertical lines define the corners of the cavity. May the reader notice that to have statistically representative average variables, these have been averaged not only in time but also in the spanwise direction. As will be shown in next sections, in the 3D-WM the pressure fluctuations that contribute to the acoustic signal quickly decay after the trailing edge. Thus in the areas of higher interest for our study, the boundary layer is resolved with $\langle y+>$ below 2 . To conclude the summary of the mesh convergence study, Figure 8 displays the ratio $\Delta / \eta$ for $3 \mathrm{D}$ WM-b results, where $\Delta$ is the mesh characteristic size and $\eta$ is the Kolmogorov scale. As can be seen, the $\Delta / \eta$ values are small enough to ensure that the turbulent structures are captured with this mesh, at least in the areas adjacent to the walls with higher contribution to the acoustic signal. 


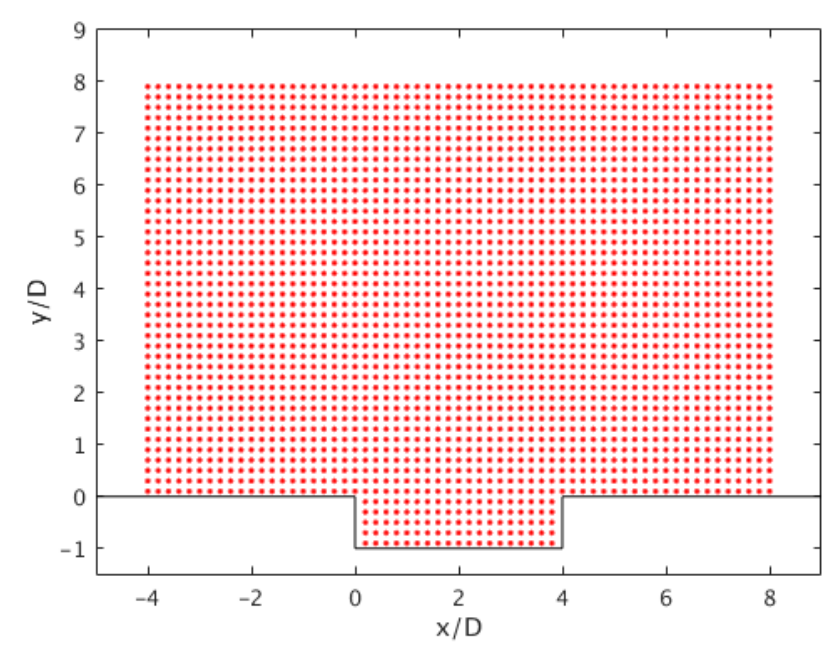

Figure 9. Observer points in the acoustic domain.

\section{Curle post-processing}

Note that in order to evaluate the surface integral of Eq.7, the pressure over the solid wall has to be stored for every time step. In the case of the two-dimensional simulation, the pressure distribution has been assumed constant in $z$ direction. Similarly, for the three-dimensional simulation the pressure has been stored for the section computed in the CFD simulation and it has been assumed to be the same for all its shifted planes. The surface integral is extended along the spanwise direction until the contribution of a new plane is negligible. Regarding the evaluation of each term of the integral in the retarded time $\tau=t-r / a_{0}$, a linear temporal interpolation has been used. The calculation of the integral is made every $\Delta t=0.1 T U$ for a total time range $T=100 T U$, where TU stands for time units $\left(\frac{t U_{\infty}}{D}\right)$. Finally, a set of 2535 acoustic observers are distributed in a mesh around the cavity (see. Fig. 9) located at plane $z / D=0$. The acoustic pressure obtained from the Curle postprocess will be calculated for the location of these observers. An observer is located every $(\Delta x, \Delta y) / D=(0.2,0.2)$.

\section{Results}

\section{Flow Field Results}

As expected from [24], the SL and WM oscillation modes are significantly different. Figure 10 displays instantaneous pressure maps for all the cases. It can be seen that for the SL mode there is a weak recirculation at the right bottom corner of the cavity generated by the impingement on the trailing edge of the vortex created in the shear layer. This mode is very similar in the two-dimensional and three-dimensional simulations, as only a very small three-dimensional structure of one unit length appears inside the cavity. This structure can be seen by means of $\mathcal{Q}$-isocontours in Figure 11, where $\mathcal{Q}$ is the second invariant of the velocity gradient tensor defined as [37]:

$$
\mathcal{Q}=-\frac{1}{2} \frac{\partial u_{i}}{\partial x_{j}} \frac{\partial u_{j}}{\partial x_{i}} .
$$

In WM the flow behaves in a completely different manner due to the higher velocity near the wall at the leading
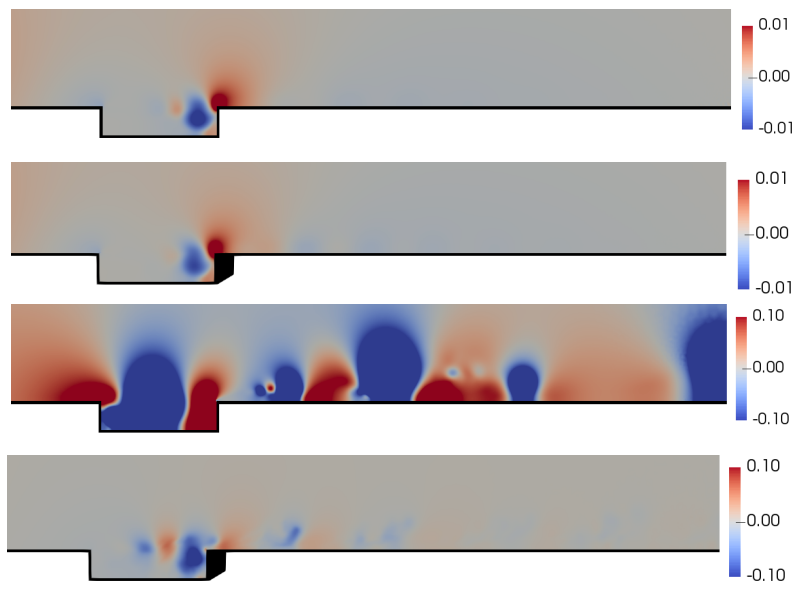

Figure 10. From top to bottom: instantaneous pressure map for 2D-SL and 3D-SL, 2D-WM and 3D-WM. For both scales the zero pressure corresponds to the pressure in the non-disturbed part of the domain.

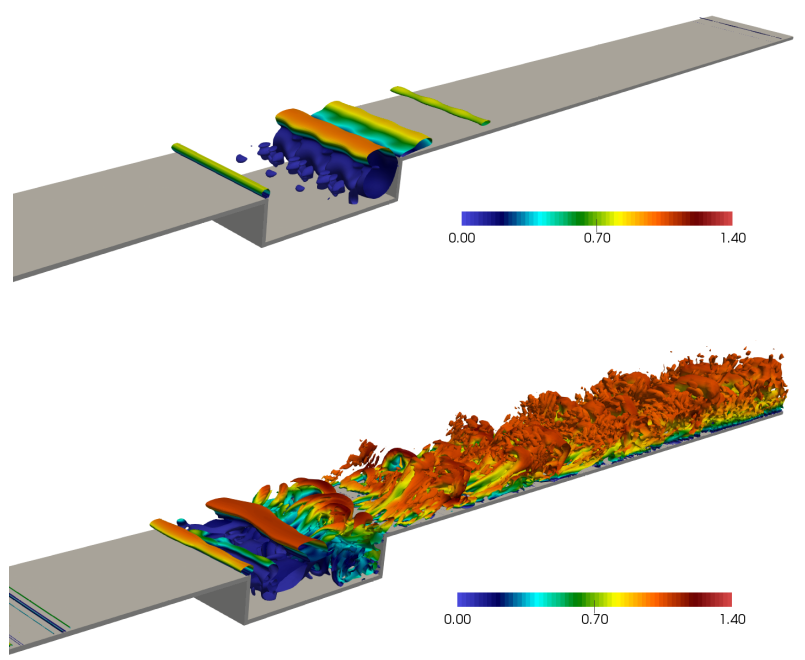

Figure 11. $\mathcal{Q}$-isocontours coloured by velocity magnitude. Top: 3D-SL. Bottom: 3D-WM.

edge of the cavity (the boundary layer thickness is smaller in WM than in SL). Therefore, the vortex shed from the leading edge is more energetic, eventually leading to its break up into smaller vortices as a consequence of the vortex stretching mechanism [38]. Hence, the 3D-WM presents more complicated structures inside the cavity and a turbulent boundary layer is developed along the downstream wall (see Fig. 11).

However, if a two-dimensional flow is assumed in WM, the results are very different and the fluid dynamics matches the description given in $[25 ; 27]$. The vortex detached from the leading edge of the cavity starts to pull fluid from the cavity generating a new growing vortex at the bottom left corner, which moves downstream until the trailing edge, where it is forced out by the one generated at the next period. Then, the vortical structures formed in the cavity edge travel downstream almost unaltered and attached to the wall of the domain (see Fig. 10).

In order to determine if the oscillations of the SL mode are sustained without the acoustic feedback, absent in 


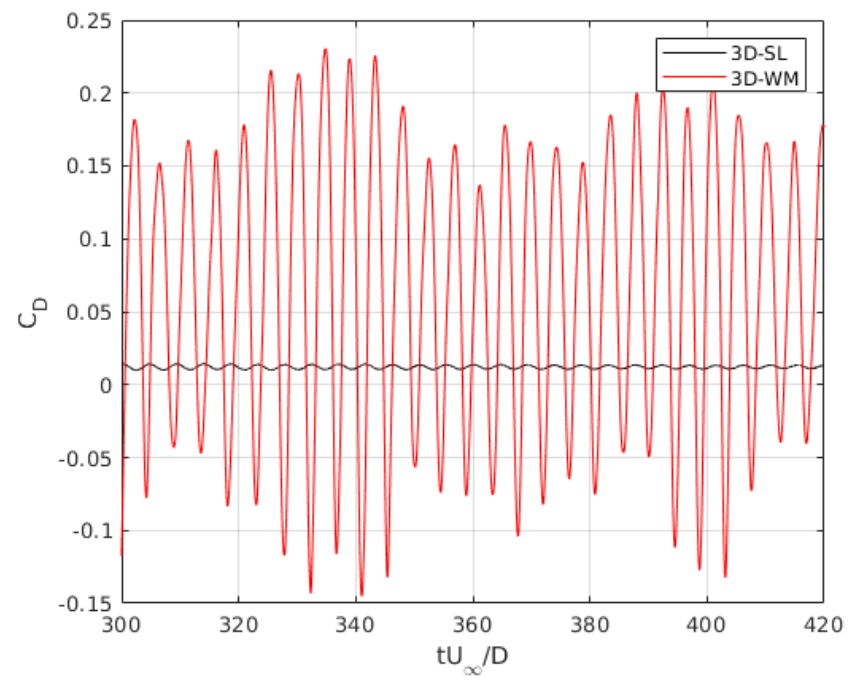

Figure 12. $C_{D}$ time evolution for 3D-SL and 3D-WM.

\begin{tabular}{|c|c|c|}
\hline Case & $C_{D}$ & $S t_{L}$ \\
\hline 2D-SL & 0.011 & 0.876 \\
\hline 2D-WM & 0.403 & 0.232 \\
\hline 3D-SL & 0.011 & 0.871 \\
\hline 3D-WM & 0.066 & 0.891 \\
\hline
\end{tabular}

Table 2. Time averaged value of drag coefficient and $S t_{L}$.

incompressible Navier-Stokes equations, the drag evolution has been represented in Fig.12. The drag force is very weak in SL, as expected from the small pressure values already shown in Fig. 10. While it is not clear if the oscillations will eventually vanish completely, they already decayed about an order of magnitude before $\frac{t U_{\infty}}{D}=300$.Runs where the boundary layer is developed during 10D, 11D and 12D (cases 3D-SL-10,3D-SL-11 and 3D-SL-12, respectively) before the leading edge of the cavity (values of $L / \theta \leq 75$ ) have been compared (see Fig.13). The results obtained in 3D-SL-11 also exhibit the decay of the oscillations while 3D-SL-10, which roughly correspond to the transition between SL and WM, does not. However, a precise determination of the transition point and mechanism are out of the scope of the present work. On the other hand, preliminary results obtained with a compressible model show self-sustained oscillations in SL mode, and this confirms the importance of the acoustic waves in the SL mode and not in the WM oscillating mode [25]. A full compressible model has to be used to describe the physics of the SL mode. Otherwise, due to the quick oscillations decay, the instant where the SL flow fields are analysed is relevant. In our case, the pressure and $\mathcal{Q}$ maps presented correspond to $T U \approx 400$, while the acoustic postprocessing was carried out between $T U=300$ and $T U=$ 400 .

Continuing with the drag coefficient, Table 2 collects its time averaged value and $S t_{L}$.

Values in Table 2 have been compared with Rossiter oscillation frequencies for an open cavity [39]. Rossiter obtained an empirical equation predicting the oscillation frequencies of the cavity:

$$
S t_{L}=\frac{m-\gamma}{\left(\frac{1}{K}+M\right)}
$$

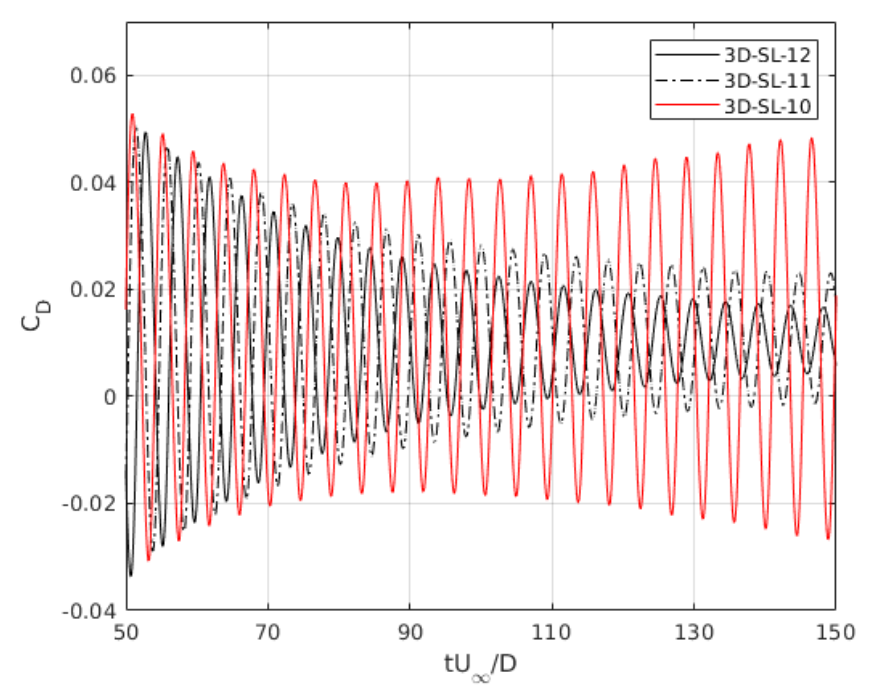

Figure 13. $C_{D}$ time evolution for 3D-SL-10, 3D-SL-11 and 3D-SL-12.

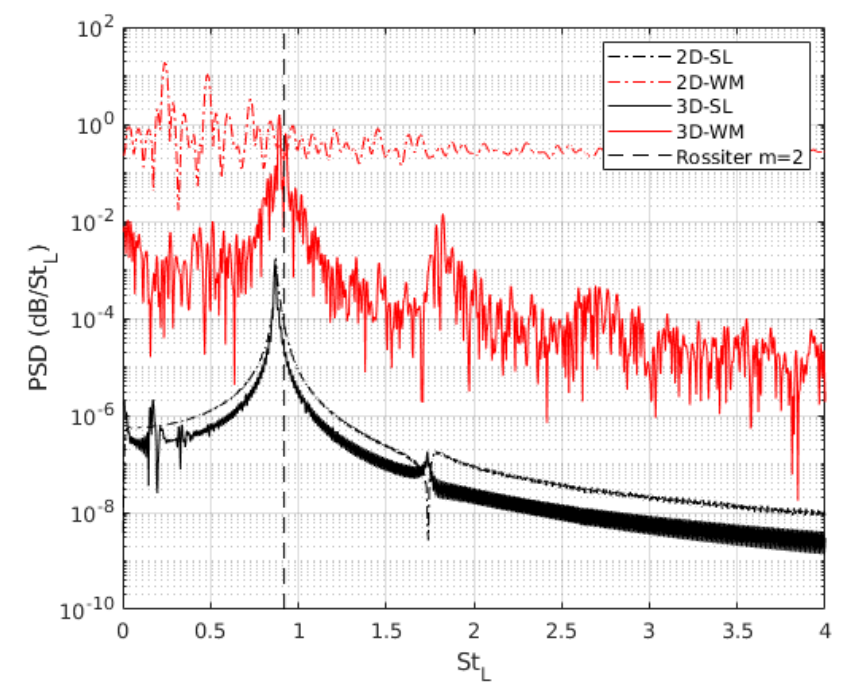

Figure 14. $S t_{L}$ spectra of $C_{D}$ for 2D-SL, 2D-WM, 3D-SL 3D-WM and Rossiter frequency for $\mathrm{m}=2$.

where $m=1,2,3, \ldots$. The experimental values for $\gamma$ and $K$ derived for $L / D=4$ were $\gamma=0.25$ and $K=0.57$. Using Eq.14, the predicted second frequency stage for $M=0.15$ using Rossiter's formula is 0.919 , a value which is in good agreement with the results shown in Table 2 for 2D-SL, 3D-SL and 3D-WM. The frequencies and mean values of $C_{D}$ obtained in 2D-WM are in agreement with other works addressing the problem with the same numerical approach [27; 18]. However, the results do not match the empirical values since they lack the vortex stretching mechanism. Hence, when the cavity is oscillating in WM, the simulation should take into account the full three-dimensional equations in order to correctly capture the behaviour of the flow, even at Reynolds numbers close to the transition to threedimensional flow.

In Fig.14, the spectra of $C_{D}$ for the different simulations have been represented. The Rossiter oscillation frequency is the dominant (except for the 2D-WM), but due to the 


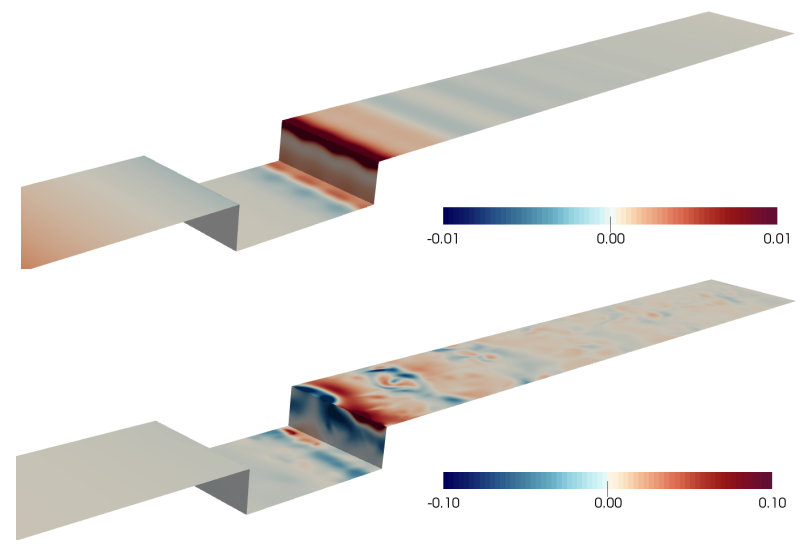

Figure 15. Instantaneous pressure on solid surface for $\mathrm{SL}$ and WM. Top: 3D-SL. Bottom: 3D-WM. For both scales the zero pressure corresponds to the pressure in the non-disturbed part of the domain.

turbulent behaviour of the fluid inside the cavity in the 3D-WM, other frequencies are also present in the spectra, highlighting again the differences between SL and WM.

Regarding to the wall pressure distributions, the instantaneous values for SL and WM can be seen in Figure 15. Their differences are significant, not only in the level but in their distribution. Figure 16 presents $\langle p\rangle$, the time average pressure distribution along the wall. Except for the 2D-WM, the mean pressure is low on the upstream cavity wall and in the beginning of the cavity. Then, for 2D-WM, the pressure rises steeply until the end of the cavity, reaching a maximum in the bottom downstream corner, followed by a minimum value at the trailing edge. In the 3D-WM, the pressure distribution is totally different: the maximum value is located at the trailing edge of the cavity. Cases 2D-SL and 3D-SL have a nearly identical distribution with a maximum value also located at the trailing edge of the cavity due to the periodic impingement of the vortex detached from the shear layer. Overall, as will be seen in the next section, the higher absolute mean pressure over the whole wall for 2D-WM will result in a much higher acoustic pressure while both shear layer cases will be very silent.

Regarding the velocities, the profiles of $\langle u\rangle$ along the $y$ axis at the streamwise positions $x / D \in\{-2,0,2,4,6,8\}$ are displayed in Fig.17. From the plot, it is noticeable that the violent vortex ejection from the cavity in 2D-WM is even affecting the boundary layer at the inlet of the cavity. Velocity profiles are also equal for 2D-SL and 3D-SL. Consequently, after all the similarities found between 2D-SL and 3D-SL, it is expected that the use of a two-dimensional simulation for SL produces correct results for this particular configuration.

\section{Acoustic Results}

The average levels of $\langle p\rangle$ shown in Figure 16 decay to zero rapidly after the cavity trailing edge. This, together with the inverse dependence with distance given by Eq.7, ensures the convergence of the surface integral for the observers located in Fig.9. For both cases, the surface of integration has been limited in the streamwise direction from $x=-6 D$ to $x=23 D$ (avoiding the buffer zone) and in the spanwise direction from $z=-30 D$ to $z=30 D$, assuming that the signal is periodic in $z$.

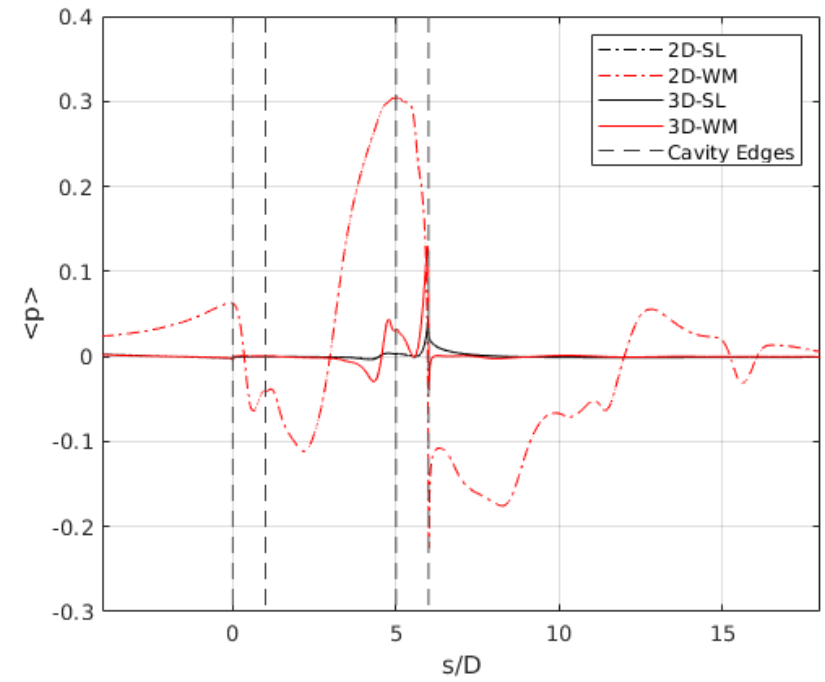

Figure 16. $\langle p>$ profile along the wall for 2D-SL, 2D-WM, 3D-SL and 3D-WM. The discontinuous vertical lines define the corners of the cavity.

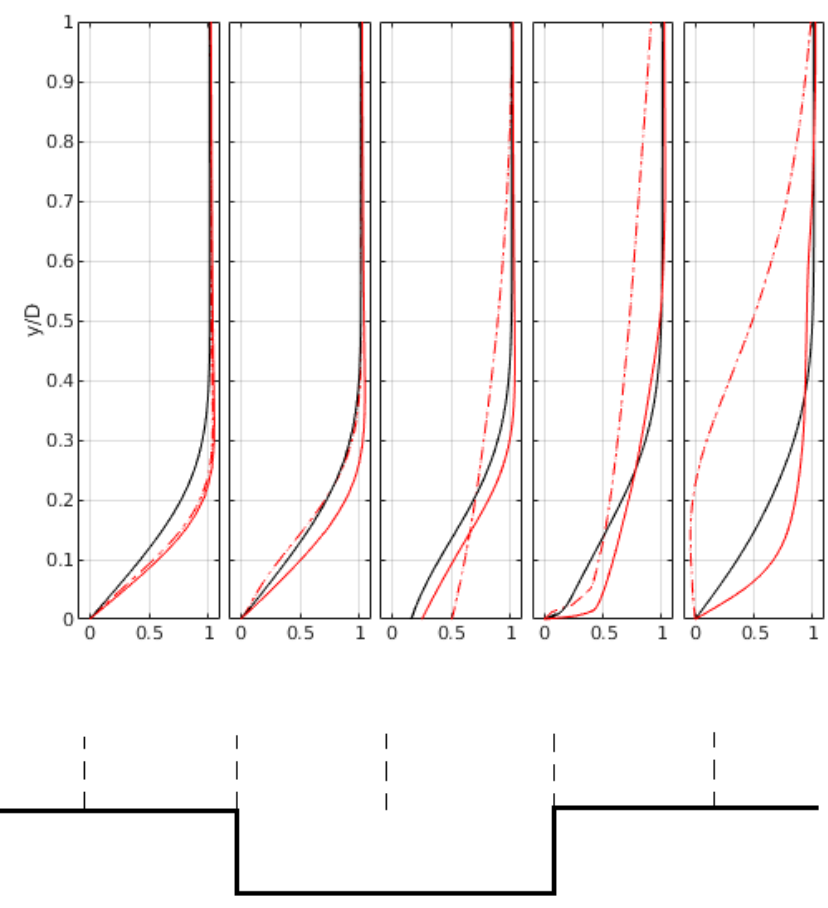

Figure 17. From left to right: $\langle u\rangle$ profiles at $x / D \in\{-2,0,2,4,6\}$ for 2D-SL (-.), 2D-WM (-.), 3D-SL (-) and 3D-WM (-).

As aforementioned, many studies have been devoted to this configuration assuming two-dimensional flow [18; 19; 20] and, under the same assumption, the present results are in fair agreement with them. The results shown in Figure 18 display the OASPL obtained by Davidson and Ask in [20] for the wake mode of the cavity when $L / \theta \approx 95$. The OASPL is defined as

$$
O A S P L=20 \log _{10}\left(\frac{p_{r m s}}{p_{r e f}}\right)
$$




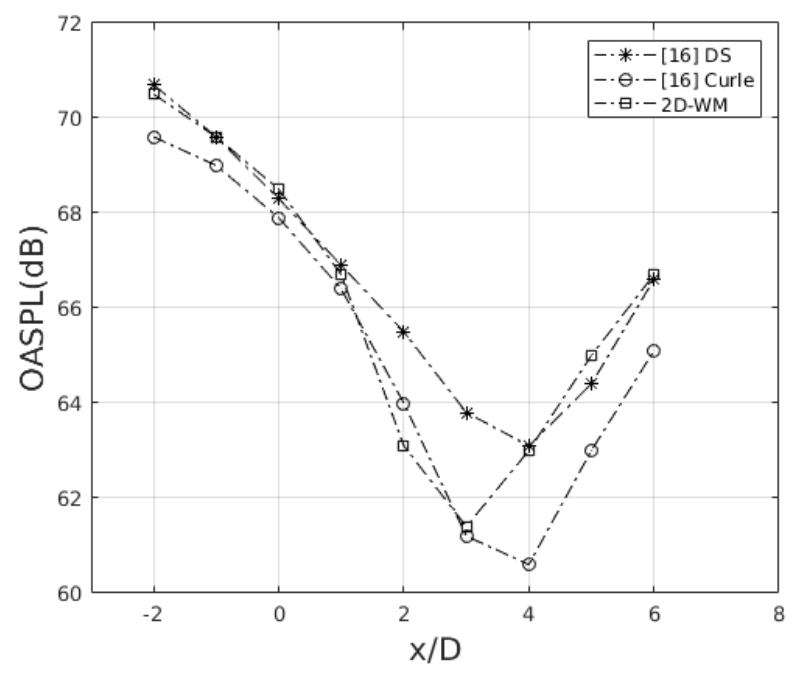

Figure 18. $O A S P L$ for observers located at $y / D=7.18$ and $x / D \in\{-2,-1,0,1,2,3,4,5,6\}$ for 2D-WM and reference [20].

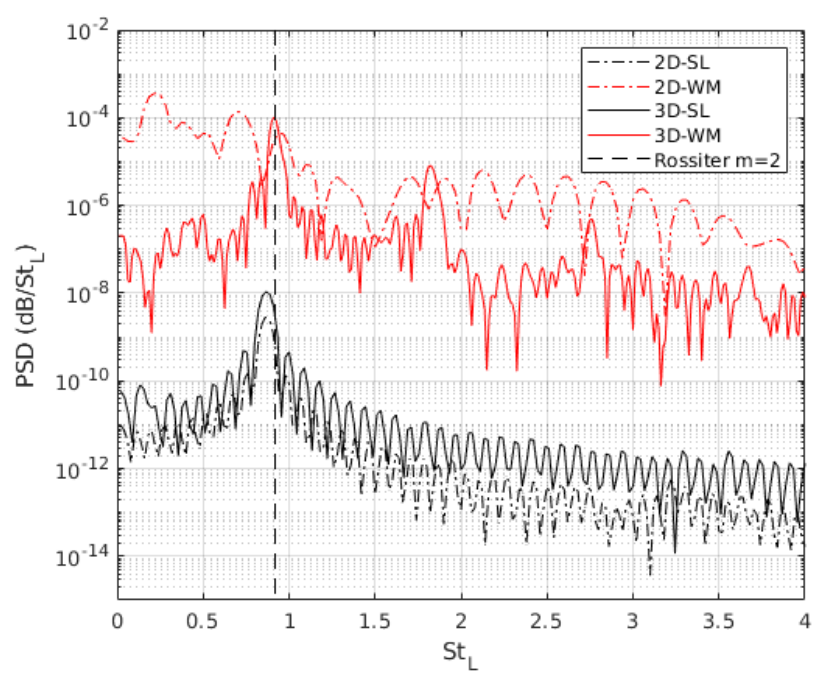

Figure 19. Frequency spectra at $(x / D, y / D)=(0,6.9)$ for 2D-SL, 2D-WM, 3D-SL and 3D-WM.

where $p_{\text {ref }}=\sqrt{\rho_{\infty} a_{0} \cdot 10^{-12}}$ and $p_{r m s}$ is the root mean square of the acoustic pressure.

The differences could be due to the different boundary layer development at the inlet of the cavity or different integration regions for the Curle integral.

Figure 19 presents the frequency spectra for the calculated acoustic signal at $(x, y)=(0 D, 6.9 D)$. The Power Spectral Density (PSD) has been calculated using the Lomb-Scargle algorithm $[40 ; 41]$. As for the shear layer mode there is one predominant frequency at $f \frac{D}{U_{\infty}}=0.240$, related with the shear layer vortex impingement. For 2D-WM, most of the energy is contained in smaller frequencies. The spectra for 3D-WM shows that there are other frequencies apart from the shedding frequency.

Figure 20 shows the OASPL for the map described by the observers in Figure 9. The 3D-WM OASPL map shows that the acoustic waves originate at the cavity trailing area, both inside and outside the cavity. The 2D-WM has a signal level much higher (about 20dB) but a similar distribution. While the SL results omit the acoustic feedback (as discussed before), it is worth noting that they are quite different, both in origin and directivity of noise.

Figure 21 shows the directivity pattern for observers located at distance 6 from the centre of the cavity, $(x, y)=$ $(2 D, 0 D)$. While the SL mode radiates more sound to the centre of the domain, the WM mode shows a high radiation upwards and downwards with a noticeable minimum at $\alpha \approx$ $70^{\circ}$, being $\alpha$ the counterclockwise angle taken from the downstream wall.

\section{Conclusions}

Numerical simulations of the sound radiated by a threedimensional infinite open cavity with aspect ratio $L / D=4$ at Reynolds number based on the cavity depth of $R e_{D}=$ 1500 and Mach number of $M=0.15$ have been performed for the two oscillation modes originally described by Gharib and Roshko [23]: i) shear layer mode (SL) and ii) wake mode (WM). The onset of one mode or the other depends on the boundary layer thickness arriving at the inlet of the domain. For these parameters the transition occurs at $\frac{L}{\theta} \approx 75$ [24]. To trigger them, values of $\frac{L}{\theta} \approx 67$ and $\frac{L}{\theta} \approx 84$ have been imposed. The acoustic method used is the Curle integral. The evaluation of the acoustic sources appearing in Curle's formulation is done as a post-process of a time-accurate flow simulation. To do so, two different approaches have been addressed: i) two-dimensional incompressible simulation and ii) three-dimensional incompressible simulation. Space and time resolutions were enough to solve all the relevant scales without the aid of sub-grid scale models.

The results obtained after a mesh convergence study show that the SL mode and the WM flow results are significantly different. The shear layer mode displays a two-dimensional behaviour while the wake mode shows three-dimensional structures as expected for a Reynolds number above $R e_{D} \approx$ 1200. The vortex stretching mechanism, responsible for the enhancement of vorticity and the transport of energy towards the smaller scales, is not present in the two-dimensional wake mode results and therefore the vortices appearing in the twodimensional simulation do not vanish. As a consequence, the pressure value over the wall changes completely, as well as the drag and other flow parameters. The drag fundamental frequency is in good agreement with published experimental data, except for the 2D-WM. In agreement with [25], the absence of acoustic feedback in incompressible flow model, results in decaying oscillations for the SL mode, that are not realistic. However, the incompressible flow model predicts realistic sustained oscillations for the WM.

Respect to the acoustic results, the two-dimensional simulations for the WM are in fair agreement with previous studies. However, for this mode the three-dimensional results are about an order of magnitude different. Thus, a twodimensional simulation would predict a nonphysical high radiation. The comparison of the directivity pattern between the SL mode and the WM shows a completely different behaviour: the SL mode radiates mostly to the centre of the domain while this direction corresponds to a well defined zone with hardly any noise propagation. 

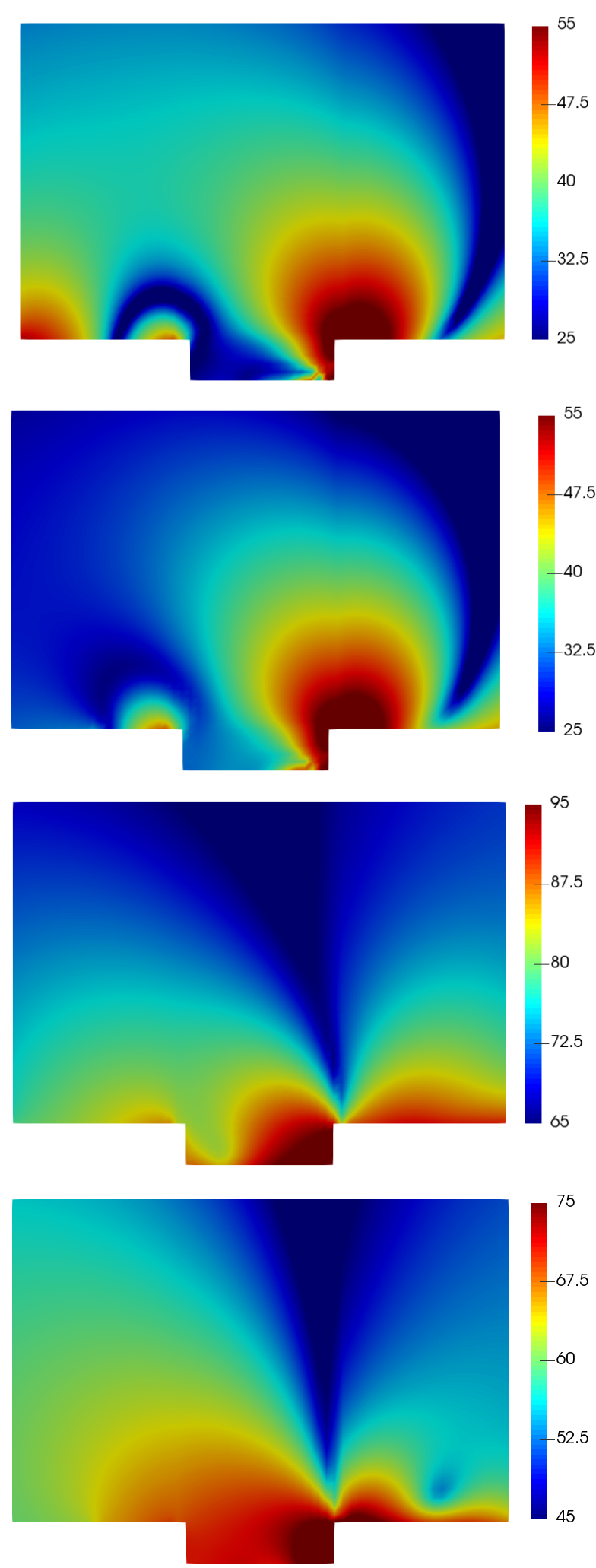

Figure 20. From top to bottom: OASPL map for 2D-SL and 3D-SL, 2D-WM and 3D-WM.

Future work will be focused on: (i) study the SL mode with direct simulations, to clarify the role of the three-dimensional fluctuations, (ii) compare Curle acoustic maps with a direct simulation for the WM in order to precisely determine the regions where the Curle's method yields accurate values and (iii) study the influence of higher Reynolds numbers and different aspect ratios between length and depth of the cavity.

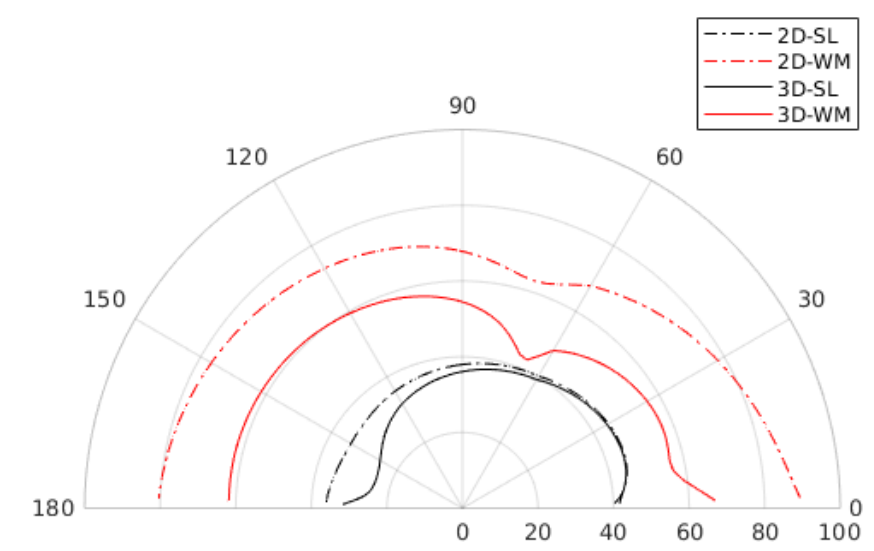

Figure 21. OASPL at observers located at distance 4 from the centre of the cavity, $(x, y)=(2 D, 0)$, for 2D-SL, 2D-WM, 3D-SL and 3D-WM.

\section{Acknowledgements}

The work of Rocio Martin was carried out within the framework of an Industrial Doctorate project from the government of Catalonia and was sponsored by the Agència de Gestió d'Ajuts Universitaris i de Recerca (AGAUR) and the automotive company SEAT S.A (2016 DI068). The work of Manel Soria has been partially supported by the Spanish Ministry (MEC) project FIS2016-77849R. The results were obtained using LaPalma (FI-2018-3-0007) and MareNotrum IV (FI-2019-1-0016) supercomputers of Red Española de Surpercomputación and computing resources of the federal collective usage center Complex for Simulation and Data Processing for Mega-science Facilities at NRC Kurchatov Institute, http://ckp.nrcki.ru/.

\section{References}

[1] Dobrzynski W, Chow LC, Smith M et al. Experimental assessment of low noise landing gear component design. International Journal of Aeroacoustics 2010; 9(6): 763-786.

[2] Souliez FJ, Long LN, Morris PJ et al. Landing gear aerodynamic noise prediction using unstructured grids. International Journal of Aeroacoustics 2002; 1(2): 115-135.

[3] Shur ML, Spalart PR and Strelets MK. Noise prediction for increasingly complex jets. part i: Methods and tests. International journal of aeroacoustics 2005; 4(3): 213-245.

[4] Khavaran A, Kenzakowski DC and Mielke-Fagan AF. Hot jets and sources of jet noise. International Journal of Aeroacoustics 2010; 9(4-5): 491-532.

[5] Hartmann M, Ocker J, Lemke T et al. Wind noise caused by the side-mirror and a-pillar of a generic vehicle model. In 18th AIAA/CEAS Aeroacoustics Conference (33rd AIAA Aeroacoustics Conference). p. 2205.

[6] Khalighi B, Snegirev A, Shinder J et al. Simulations of flow and noise generated by automobile outside rear-view mirrors. International Journal of Aeroacoustics 2012; 11(1): 137-156.

[7] Pérot F, Gloerfelt X, Bailly $\mathrm{C}$ et al. Numerical prediction of the noise radiated by a cylinder. In 9th AIAA/CEAS Aeroacoustics Conference and Exhibit 2003. p. 3240.

[8] Yao HD, Davidson L and Eriksson LE. Noise radiated by lowreynolds number flows past a hemisphere at ma=0.3. Physics of Fluids 2017; 29(7): 076102. 
[9] Erbig L and Maihöfer M. A hybrid rans/les for automotive gap noise simulations. In 25th AIAA/CEAS Aeroacoustics Conference. p. 2445.

[10] Wagner C, Hüttl T and Sagaut P. Large-eddy simulation for acoustics, volume 20. Cambridge University Press, 2007.

[11] Lighthill MJ. On sound generated aerodynamically. i. general theory. In Proceedings of the Royal Society of London A: Mathematical, Physical and Engineering Sciences 1952, volume 211. The Royal Society, pp. 564-587.

[12] Curle N. The influence of solid boundaries upon aerodynamic sound. In Proceedings of the Royal Society of London A: Mathematical, Physical and Engineering Sciences 1955, volume 231. The Royal Society, pp. 505-514.

[13] Williams JF and Hawkings DL. Sound generation by turbulence and surfaces in arbitrary motion. Philosophical Transactions of the Royal Society of London A: Mathematical, Physical and Engineering Sciences 1969; 264(1151): 321342.

[14] Lai H and Luo KH. A three-dimensional hybrid les-acoustic analogy method for predicting open-cavity noise. Flow, turbulence and Combustion 2007; 79(1): 55-82.

[15] Mancini S, Kolb A, Gonzalez-Martino I et al. Effects of wall modifications on pressure oscillations in high-subsonic and supersonic flows over rectangular cavities. In 25th AIAA/CEAS Aeroacoustics Conference. p. 2692.

[16] Kuhn T. Zonal large eddy simulation of active open cavity noise using a high order discontinuous galerkin method. In 25th AIAA/CEAS Aeroacoustics Conference. p. 2465.

[17] Hardin JC and Pope DS. Sound generation by flow over a two-dimensional cavity. AIAA journal 1995; 33(3): 407-412.

[18] Larsson J, Davidson L, Olsson M et al. Aeroacoustic investigation of an open cavity at low mach number. AIAA journal 2004; 42(12): 2462-2473.

[19] Ask J and Davidson L. An acoustic analogy applied to the laminar upstream flow over an open $2 \mathrm{~d}$ cavity. Comptes Rendus Mecanique 2005; 333(9): 660-665.

[20] Ask J and Davidson L. Sound generation and radiation of an open two-dimensional cavity. AIAA J 2009; 47(6): 13371349.

[21] Bres GA and Colonius T. Three-dimensional instabilities in compressible flow over open cavities. Journal of Fluid Mechanics 2008; 599: 309-339.

[22] Faure TM, Adrianos P, Lusseyran F et al. Visualizations of the flow inside an open cavity at medium range reynolds numbers. Experiments in Fluids 2007; 42(2): 169-184.

[23] Gharib M and Roshko A. The effect of flow oscillations on cavity drag. Journal of Fluid Mechanics 1987; 177: 501-530.

[24] Colonius T, Basu A and Rowley C. Numerical investigation of the flow past a cavity. In 5th AIAA/CEAS Aeroacoustics Conference and Exhibit 1999. p. 1912.

[25] Rowley CW, Colonius T and Basu AJ. On self-sustained oscillations in two-dimensional compressible flow over rectangular cavities. Journal of Fluid Mechanics 2002; 455: 315-346.

[26] Yao HD, Davidson L, Eriksson LE et al. Surface integral analogy approaches for predicting noise from 3d high-lift lownoise wings. Acta Mechanica Sinica 2014; 30(3): 326-338.

[27] Larsson J, Davidson L, Olsson M et al. Aeroacoustic investigation of an open cavity at low mach number. AIAA journal 2004; 42(12): 2462-2473.

[28] Farassat F and Myers M. Extension of kirchhoff's formula to radiation from moving surfaces. Journal of Sound and Vibration 1988; 123(3): 451-460.

[29] Kirchhoff G. Zur theorie der lichtstrahlen. Annalen der Physik 1883; 254(4): 663-695.

[30] Michel U. History of acoustic beamforming. In 1st. Berlin Beamforming Conference 2006.

[31] Vázquez M, Houzeaux G, Koric S et al. Alya: Multiphysics engineering simulation toward exascale. Journal of Computational Science 2016; 14: 15-27.

[32] Charnyi S, Heister T, Olshanskii MA et al. On conservation laws of navier-stokes galerkin discretizations. Journal of Computational Physics 2017; 337: 289-308.

[33] Codina R. Pressure stability in fractional step finite element methods for incompressible flows. Journal of Computational Physics 2001; 170(1): 112-140.

[34] Jofre L, Lehmkuhl O, Ventosa $\mathrm{J}$ et al. Conservation properties of unstructured finite-volume mesh schemes for the navier-stokes equations. Numerical Heat Transfer, Part B: Fundamentals 2014; 65(1): 53-79.

[35] Lehmkuhl O, Houzeaux G, Avila M et al. A low dissipation finite element scheme for the large eddy simulation on complex geometries. In $19 \mathrm{~h}$ International Conference on Finite Elements in Flow Problems-FEF 2017.

[36] Capuano F, Coppola G, Rández L et al. Explicit rungekutta schemes for incompressible flow with improved energyconservation properties. Journal of Computational Physics 2017; 328: 86-94.

[37] Jeong $J$ and Hussain F. On the identification of a vortex. Journal of fluid mechanics 1995; 285: 69-94.

[38] Tennekes H and Lumley JL. A first course in turbulence. MIT press, 1972.

[39] Rossiter J. Wind tunnel experiments on the flow over rectangular cavities at subsonic and transonic speeds. Technical report, Ministry of Aviation; Royal Aircraft Establishment; RAE Farnborough, 1964.

[40] Lomb NR. Least-squares frequency analysis of unequally spaced data. Astrophysics and space science 1976; 39(2): $447-462$.

[41] Scargle JD. Studies in astronomical time series analysis. iistatistical aspects of spectral analysis of unevenly spaced data. The Astrophysical Journal 1982; 263: 835-853. 